\title{
Sustained Achromobacter Xylosoxidans Bacteremia in a Patient with Adenocarcinoma of the Colon
}

\author{
Matt Enriquez, MS IV, Andi Favini MS IV, Kevin Curl, MD
}

\section{INTRODUCTION}

Achromobacter xylosoxidans is a rare cause of bacteremia; however patients with underlying illness, especially malignancies, are at increased risk of infection. Antibiotic therapy against this pathogen can be difficult owing to its inherent resistance to multiple common antibiotics.

\section{CASE PRESENTATION}

A 69-year-old male with adenocarcinoma of the colon status post right hemicolectomy and chemotherapy presented with fever and fatigue 10 days following endoscopic retrograde cholangiopancreatography (ERCP) with biliary stent placement for new liver metastases. The patient had undergone partial hemicolectomy and had completed 12 cycles of adjuvant FOLFOX (leucovorin, 5-fluorouracil, and oxaliplatin) at the time of initial diagnosis. Two years following the completion of chemotherapy, the patient was found to have obstructive jaundice and new liver metastases on CT scan. He underwent an ERCP procedure with biliary stenting for palliation and was discharged home.

Ten days following the procedure, the patient presented with complaints of fever to $102^{\circ} \mathrm{F}$ and fatigue. Vital signs revealed a fever of $101.3 \circ \mathrm{F}$ but were otherwise unremarkable. Physical examination revealed improvement of his jaundice, normal cardiopulmonary examination, and right upper quadrant tenderness. Despite four days of vancomycin and piperacillintazobactam, the patient continued to be febrile to $102.5^{\circ} \mathrm{F}$.

\section{DIFFERENTIAL DIAGNOSIS}

The medical team remained concerned about infectious etiologies, including cholangitis, bacteremia, and endocarditis. Non-infectious causes of his fever were

Table 1. Antibiotic sensitivities (represented as percent susceptible) of Achromobacter xylosoxidans in several case reports and the current patient's susceptibilities.

\begin{tabular}{|c|c|c|c|}
\hline Antibiotic & $\begin{array}{l}\text { Turel O, et al. } \\
\text { (34 isolates) }\end{array}$ & $\begin{array}{l}\text { Glupczynski Y, et al. } \\
\text { ( } 37 \text { isolates) }\end{array}$ & Current Patient \\
\hline \multicolumn{4}{|l|}{ Antibiotic } \\
\hline Trimethoprim-Sulfamethoxazole & 100 & 67 & Unknown \\
\hline Meropenem & 100 & 100 & Susceptible \\
\hline Piperacillin-Tazobactam & 91 & 91 & Resistant \\
\hline Ciprofloxacin & 82 & 0 & Susceptible \\
\hline Ceftazidime & 82 & 81 & Resistant \\
\hline Cefepime & 15 & 0 & Unknown \\
\hline Gentamicin & 0 & 0 & Intermediate \\
\hline
\end{tabular}


also considered, such as post-ERCP pancreatitis, deep venous thrombosis, and malignancy-related fever.

\section{OUTCOME AND FOLLOW-UP}

Routine laboratory studies were unremarkable, including serum amylase and lipase. Right upper quadrant ultrasound and CT scan of the abdomen and pelvis did not show any visible infectious source. Transthoracic echocardiogram was not consistent with endocarditis. Lower extremity Dopplers were negative, and while upper extremity Dopplers revealed no deep venous thrombosis, they did show a right cephalic vein clot. Blood cultures drawn on admission were found to be positive for gram-negative bacilli on hospital day three. Subsequent blood cultures drawn after the initiation of broad-spectrum antibiotics were also positive for gram-negative bacilli. Prior to speciation, the Infectious Disease team recommended discontinuing vancomycin and switching the piperacillin-tazobactam to meropenem. On hospital day number seven, the patient became afebrile and blood culture speciation revealed Achromobacter xylosoxidans with sensitivities listed in Table 1. Blood cultures drawn after the adjustment of antibiotic therapy to meropenem remained negative. It was believed that the patient had a transient bacteremia of Achromobacter xylosoxidans related to the ERCP that had seeded his cephalic vein thrombus, leading to persistent bacteremia. Based on culture sensitivities, the patient's antibiotics were changed to oral ciprofloxacin to complete a four week course of antibiotics. He was also started on prophylactic enoxaparin for his cephalic vein thrombus and was discharged home.

\section{DISCUSSION}

Bacteremia due to A. xylosoxidans remains rare, yet patients with underlying illness, particularly malignancies, are at increased risk of infection. Other predisposing conditions include cardiovascular disease, renal failure, and immune suppression. ${ }^{1}$ Though A. xylosoxidans is not a typical component of endogenous human flora, it is known to inhabit aqueous environments with common sources including contaminated water and IV fluids, intravenous catheters, humidification fluids, and instrumentation utilized during surgical interventions. ${ }^{1}$
Common symptoms of $A$. xylosoxidans bacteremia include fever, fatigue, and persistence of symptoms despite broad-spectrum antibiotic therapy. A unique symptom to this presentation was right upper quadrant abdominal pain consistent with a gastrointestinal source of infection secondary to the patient's prior ERCP. Aside from gastrointestinal infections, other common sources of infection include catheter-associated infections and pneumonia.

As A. xylosoxidans bacteremia is often associated with specific predisposing conditions and sources of infection, early identification of risk factors for infection remains a crucial component of diagnosis. Initial diagnostic tests to determine if the bacteremia is due to $A$. xylosoxidans include repeating blood cultures which may demonstrate sustained gram-negative rod bacteremia. Another useful part of the diagnostic work-up includes identifying the original source of the patient's bacteremia in addition to ongoing site of infection. In this case, ultrasound served to localize a right cephalic vein clot as the probable ongoing site of infection; this clot had likely been seeded by a gastrointestinal source around the time of the ERCP procedure.

Choosing antibiotic therapy to treat A. xylosoxidans bacteremia proves challenging because of its inherent resistance to multiple antibiotics in addition to its ability to harbor and horizontally transfer resistance genes (Table 1). A proposed transfer mechanism for A. xylosoxidans has been shown to potentially involve integrons, R plasmids, and insertion sequences. ${ }^{2-4}$

A. xylosoxidans remains an important emerging cause of nosocomial and community-acquired infections because of its resistance to multiple antibiotics. Although individual patterns vary, most strains of A. xylosoxidans are resistant to aminoglycosides and quinolones. ${ }^{2}$ The majority of isolates have demonstrated susceptibility to tazobactam, imipenem and meropenem, but as demonstrated in Table 1, this patient harbored a strain that was also resistant to piperacillin-tazobactam. Aggressive measures to sterilize hospital solutions and equipment, as well as to enforce policies to prevent antibiotic misuse, should be employed to prevent future cases of Achromobacter xylosoxidans bacteremia and to quell the propagation of drug resistance in this pathogen. 


\section{KEY POINTS}

This case serves as a reminder to consider A. xylosoxidans when treating patients with underlying illnesses, particularly malignancies, who have persistent gram-negative bacteremia despite therapy with common broad-spectrum antibiotics.

\section{REFERENCES}

1. Duggan J, Goldstein S, Chenoweth C, Kauffman C, Bradley S. Achromobacter xylosoxidans bacteremia: report of four cases and review of the literature. Clin. Infect. Dis. 1996; 23(3): 569-76.

2. Traglia G, Almuzara M, Merkier A, Adams C, Galanternik L, Vay C, Centron D, Ramirez M. Achromobacter xylosoxidans: an emerging pathogen carrying different elements involved in horizontal genetic transfer. Curr. Microbiol. 2012; 65(6): 673-8.

3. Turel O, Kavuncuoglu S, Hosaf E, et al. Bacteremia due to Achromobacter xylosoxidansin neonates: clinical features and outcome. Braz J Infect Dis. 2013; 17(4): 450-4.

4. Glupczynski Y, Hansen W, Freney J, Yourassowsky E. In vitro susceptibility of Alcaligenes dentrificans subsp. Xylosoxidans to 24 antimictrobial agents. Antimicrob Agents Chemother. 1988; 32(2): $276-8$ 